\title{
LOG MINIMAL MODEL PROGRAM FOR THE MODULI SPACE OF STABLE CURVES OF GENUS THREE
}

\author{
Donghoon Hyeon and Yongnam Lee
}

\begin{abstract}
We completely carry out the log minimal model program for the moduli space of stable curves of genus three with respect to the total boundary $\delta$ : We give a modular interpretation of the log canonical model for the pair $\left(\overline{\mathcal{M}}_{3}, \alpha \delta\right)$ for each $\alpha \in$ $[0,7 / 10)$ and of the birational maps between them. By using the modular description, we are able to identify all but one $\log$ canonical models $\bar{M}_{g}(\alpha), \alpha \in[0,1]$, with existing compactifications of $M_{3}$, some new and others classical, while the exception gives a new modular compactification of $M_{3}$.
\end{abstract}

\section{Introduction and Preliminaries}

In this paper, we shall carry out the $\log$ Mori program for $\bar{M}_{3}$ with respect to the total boundary $\delta$ of singular curves. That is, we shall consider the log canonical models

$$
\bar{M}_{3}(\alpha):=\text { Proj } \oplus_{m \geq 0} \Gamma\left(\bar{M}_{3}, m\left(K_{\overline{\mathcal{M}}_{3}}+\alpha \delta\right)\right)
$$

as we decrease $\alpha$ from 1 to 0 and describe these spaces and the relation between them in a concrete manner. The basic principle, credited to Brendan Hassett and Sean Keel, is that these log canonical models themselves admit modular interpretation and parametrize curves with increasingly worse singularities as $\alpha$ decreases. This principle has been demonstrated in a series of work by Hassett and the authors $[6,8,7,9]$. It will be in full display again in our work for the genus three case.

By a theorem of Cornalba and Harris, $K_{\overline{\mathcal{M}}_{g}}+\alpha \delta$ is ample for $9 / 11<\alpha \leq 1$ and $\bar{M}_{g}(\alpha)$ is isomorphic to $\bar{M}_{g}$ for $\alpha$ in that range. At $\alpha=9 / 11$, it is shown in [8] that the locus of elliptic tails gets contracted, resulting the moduli space $\bar{M}_{g}^{p s}$ of pseudostable curves of Schubert [17]. In [7], Hassett and the first named author described what happens near the second critical value $\alpha=7 / 10$ for the case $g \geq 4$. Since we shall need the statements for proving and presenting our main theorem, we reproduce them here:

Theorem. [7]

(1) There is a small contraction $\Psi: \bar{M}_{g}^{p s} \rightarrow \bar{M}_{g}(7 / 10)$ and $\bar{M}_{g}(7 / 10)$ is isomorphic to the GIT quotient $C h o w_{g, 2} / / S L_{3 g-3}$ of the Chow variety of bicanonical curves. $\Psi$ contracts the locus $Z$ of elliptic bridges;

(2) There exists a flip $\Psi^{+}:\left(\bar{M}_{g}^{p s}\right)^{+} \rightarrow \bar{M}_{g}(7 / 10)$ and $\left(\bar{M}_{g}^{p s}\right)^{+}$is isomorphic to $\bar{M}_{g}(\alpha)$ for $7 / 10-\epsilon<\alpha<7 / 10$. Moreover, $\bar{M}_{g}(\alpha)$ for $\alpha$ in that range is isomorphic to the GIT quotient $\mathrm{Hilb}_{g, 2} / / S L_{3 g-3}$ of the Hilbert scheme of bicanonical curves, and $\Psi^{+}$contracts the locus $Z^{+}$of tacnodal curves.

Received by the editors June 10, 2008. 
Here, $\mathrm{Hilb}_{g, 2}$ denotes the closure of locus of smooth curves in the Hilbert scheme of subschemes of $\mathbb{P}^{3 g-4}$ with Hilbert polynomial $P(t)=(g-1)(4 t-1)$. Chow ${ }_{g, 2}$ denotes the corresponding Chow variety. An elliptic bridge is a subcurve of genus one meeting the rest of the curve in two nodes. Abusing terminology, as in the theorem above, an elliptic bridge sometimes means a curve with such a subcurve of genus one. The proof of the above theorem in [7] for $g \geq 4$ goes through for the genus three case when combined with the input of the necessary GIT of bicanonical curves of genus three worked out in [10]. The main theorem of GIT of bicanonical curves of genus three is:

Theorem. [10] The (semi)stable points of $\mathrm{Hilb}_{3,2}$ (resp. Chow ${ }_{3,2}$ ) correspond to h(semi)stable (resp. c-(semi)stable) curves. Moreover, all strictly c-semistable points are identified in $\mathrm{Chow}_{3,2} / / S L_{6}$.

We refer to [7] for the definitions of the h- and c-(semi)stability for curves of arbitrary genus $\geq 3$, which are rather intricate. For the genus three case, they may be defined as follows: A complete curve $C$ of genus three is said to be $c$-semistable if

(1) $C$ has nodes, cusps and tacnodes as singularities;

(2) $\omega_{C}$ is ample;

(3) a genus one subcurve meets the rest of the curve in at least two points not counting multiplicity.

If $C$ is c-semistable and has no tacnode or elliptic bridge, it is c-stable. If $C$ is csemistable and has no elliptic bridge, it is said to be h-semistable. $C$ is said to be $h$-stable if it is h-semistable and has no tacnode. ${ }^{1}$

We shall denote the quotient spaces $H_{i l b}, 2 / / S L_{6}$ and $C h o w_{3,2} / / S L_{6}$ by $\bar{M}_{3}^{h s}$ and $\bar{M}_{3}^{c s}$ respectively. Our main theorem is:

Theorem 1. (1) $\bar{M}_{3}(\alpha)$ is isomorphic to $\bar{M}_{3}^{\text {hs }}$ for $17 / 28<\alpha<7 / 10$;

(2) There is a divisorial contraction $\Theta: \bar{M}_{3}^{\text {hs }} \rightarrow \bar{M}_{3}(17 / 28)$ that contracts the hyperelliptic locus, and $\bar{M}_{3}(17 / 28)$ is isomorphic to the compact moduli space $\bar{Q}:=\mathbb{P}\left(\Gamma\left(\mathcal{O}_{\mathbb{P}^{2}}(4)\right)\right) / / S L_{3}$ of plane quartics;

(3) $\bar{M}_{3}(\alpha)$ is isomorphic to $\bar{Q}$ for $5 / 9<\alpha \leq 17 / 28$;

(4) $\bar{M}_{3}(5 / 9) \simeq$ Spec $k$ and for $\alpha<5 / 9$, no Cartier multiple of $K_{\overline{\mathcal{M}}_{3}}+\alpha \delta$ has nontrivial sections.

Also, the fact that all tacnodal curves are identified in $\bar{M}_{3}^{c s}$ allows us to identify $\bar{M}_{3}^{c s}$ with the compact moduli space of plane quartics constructed by Kondo using the period domains of K3 surfaces (Proposition 3). The following diagram gives an

\footnotetext{
${ }^{1}$ For genus three curves, h-stability is equivalent to c-stability.
} 
overview of the whole program:

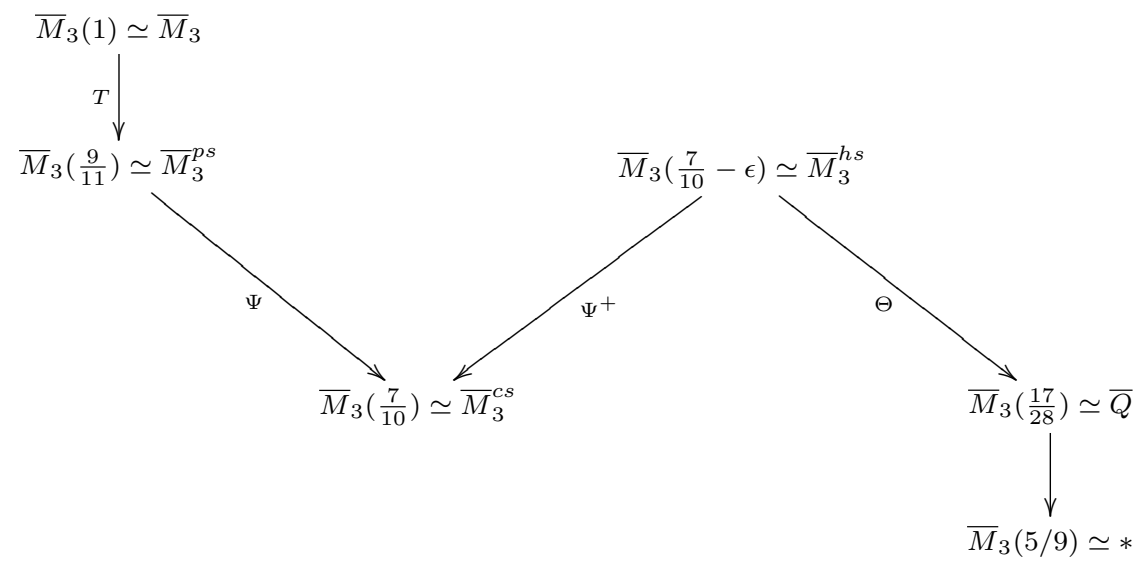

We work over an algebraically closed field of characteristic zero.

\section{Acknowledgements}

D.H. would like to thank Brendan Hassett for suggesting this problem and for many helpful conversations. He was partially supported by KIAS. He gratefully acknowledges Bumsig Kim for the hospitality and for useful conversations.

Y.L. would like to thank Shigeyuki Kondo for his explanation of his compact moduli space, Shigefumi Mori for his explanation of birational geometry, Shigeru Mukai for his valuable comments on the geometric invariant theory. This article was completed while Y.L. was visiting RIMS at Kyoto University by the JSPS Invitation Fellowship Program, and Mittag-Leffler Institute. He thanks Shigefumi Mori for the invitation and hospitality during his stay at RIMS, and Carel Faber for the invitation and hospitality during his stay at Mittag-Leffler Institute.

Both authors thank Noboru Nakayama for his valuable comments and suggestions that improved this article. The main theorem of this article was suggested to us by Brendan Hassett, and we thank him for generously sharing his ideas. After our work was completed, we learned that Sean Keel had obtained some of the results in this paper independently a few years ago. Both authors were supported by the Korea Research Foundation Grant funded by the Korea Government (MOEHRD) (KRF-2005-042-C00005).

\section{Modular interpretation of the log canonical models}

2.1. Detecting the third critical value for $\alpha$. In this section, we shall prove the first part of Theorem 1. Fix $m \gg 0$ and let $\pi: H i l b_{3,2} \rightarrow \bar{M}_{3}^{h s}=H i l b_{3,2} / / S L_{6}$ denote the quotient map, where $\mathrm{Hilb}_{3,2}$ is embedded by taking the $\mathrm{m}$ th Hilbert points $H_{i l b_{3,2}} \hookrightarrow \mathbb{P}\left(\bigwedge^{P(m)} S^{m} V\right), \operatorname{dim} V=6$, and the $S L_{6}$ action is linearized accordingly. The projective structure on $\bar{M}_{3}^{h s}$ is given by the invariant sections of $\mathcal{O}_{H_{i l b}, 2}(m)$ on $H_{i l b}$. That is, we have $\pi^{*}\left(\mathcal{O}_{\bar{M}_{3}^{h s}}(1)\right)=\mathcal{O}_{H_{i l b_{3,2}}}(m)$ and by [15, Proposition 5.16], 
it is proportional to

$$
\left(10-\frac{3}{2 m}\right) \lambda-\delta .
$$

where $\lambda$ and $\delta$ denote the determinant of the Hodge bundle and the divisor of singular curves, respectively. ${ }^{2}$ Hence for large enough $m,\left(10-\frac{3}{2 m}\right) \lambda-\delta$ descends to an ample $\mathbb{Q}$-divisor on $\bar{M}_{3}^{h s}$. It follows that $\lambda$ (and consequently, $\delta$ ) descends to a Cartier divisor since it is proportional to the difference of $\left(10-\frac{3}{2 m}\right) \lambda-\delta$ and $\left(10-\frac{3}{2(m+1)}\right) \lambda-\delta$. Let $\Lambda^{h s}$ and $\Delta^{h s}$ denote the Cartier divisors on the moduli scheme $\bar{M}_{3}^{h s}$ that pull back to $\lambda$ and $\delta$ via the birational map from $\bar{M}_{3}$ to $\bar{M}_{3}^{h s}$. They also coincide with the divisors coming from $\lambda$ and $\delta$ on the Hilbert scheme $H i l b_{3,2}$. Likewise, let $\lambda^{p s}$ and $\delta^{p s}$ denote the corresponding divisors on $\bar{M}_{3}^{p s}$. Since $T$ contracts $\delta_{1}$ and the locus of elliptic tails in $\bar{M}_{3}^{p s}$ is of codimension two, the identity $K_{\overline{\mathcal{M}}_{3}}=13 \lambda-2 \delta$ translates to $K_{\overline{\mathcal{M}}_{3}^{p s}}=13 \lambda^{p s}-2 \delta^{p s}$ on $\bar{M}_{3}^{p s}$. Since $\bar{M}_{3}^{p s}$ and $\bar{M}_{3}^{h s}$ are isomorphic away from loci of codimension $\geq 2$, it further carries over to $\bar{M}_{3}^{h s}$ to give $K_{\bar{M}_{3}^{h s}}+1 / 2 \bar{H}^{h s}=$ $13 \Lambda^{h s}-2 \Delta^{h s}$, where $\bar{H}^{h s}$ is the divisor of hyperelliptic curves. Since (1) is ample on $\bar{M}_{3}^{h s}$ for large enough $m, K_{\bar{M}_{3}^{h s}}+1 / 2 \bar{H}^{h s}+(7 / 10-\epsilon) \Delta^{h s}$ is ample for small enough $\epsilon$, being a positive rational multiple of (1).

Proposition 1. $K_{M_{3}^{h s}}+\frac{1}{2} \bar{H}^{h s}+\frac{17}{28} \Delta^{h s}$ is nef and big on $\bar{M}_{3}^{h s}$ and has a unique extremal ray generated by hyperelliptic curves.

Proof. Note that the divisor $K_{M_{3}^{h s}}+\frac{1}{2} \bar{H}^{h s}+\frac{17}{28} \Delta^{h s}$ in question is proportional to $28 \Lambda^{h s}-3 \Delta^{h s}$, which also coincides with (the proper transform of) the Moriwaki divisor [13] $A=28 \lambda-3 \delta_{0}-8 \delta_{1}$. By [13, Theorem B], Moriwaki divisor positively intersects any curve not contained in the boundary $\Delta^{\text {hs }}$, and it follows that the divisor is big.

Since the hyperelliptic locus is equal to $9 \lambda-\delta_{0}-3 \delta_{1}$ on $\bar{M}_{3}$, the corresponding locus $\bar{H}^{h s}$ on $\bar{M}_{3}^{h s}$ is equal to $9 \Lambda^{h s}-\Delta^{h s}$ and we have

$$
28 \Lambda^{h s}-3 \Delta^{h s}=\left(10 \Lambda^{h s}-\Delta^{h s}\right)+2\left(9 \Lambda^{h s}-\Delta^{h s}\right)=\left(10 \Lambda^{h s}-\Delta^{h s}\right)+2 \bar{H}^{h s} .
$$

The divisor $10 \Lambda^{h s}-\Delta^{h s}$ is nef, since it is proportional to $K_{M_{3}^{h s}}+\frac{1}{2} \bar{H}^{h s}+\frac{7}{10} \Delta^{h s}$ which is a limit of ample divisors. It follows that to show the nefness of $28 \Lambda^{h s}-3 \Delta^{h s}$, it suffices to prove that the divisor non-negatively intersects with curves in $\bar{H}^{h s}$. In fact, we shall prove that $28 \Lambda^{h s}-3 \Delta^{h s}$ is trivial on $\bar{H}^{h s}$.

\footnotetext{
${ }^{2}$ Note our abuse of notation as $\lambda$ and $\delta$ are also used to denote the corresponding divisor classes on the moduli stack $\overline{\mathcal{M}}_{3}$. Moreover, if $\sigma$ is a divisor class on the moduli stack, then 'the divisor $\sigma$ on the moduli space' means the corresponding divisor class on the moduli space through $\operatorname{Pic}_{\mathbb{Q}}\left(\overline{\mathcal{M}}_{3}\right)=$ $\operatorname{Pic}_{\mathbb{Q}}\left(\bar{M}_{3}\right)$. This significantly simplifies our presentation: For instance, $\delta$ on $\bar{M}_{3}$ means $\Delta_{0}+\frac{1}{2} \Delta_{1}$ and $K_{\overline{\mathcal{M}}_{3}}$ on $\bar{M}_{3}$ means $K_{\bar{M}_{3}}+\frac{1}{2} \Delta_{1}+\frac{1}{2} \bar{H}$ where $\bar{H}$ is the divisor of hyperelliptic curves. These are just ramification formulae.
} 


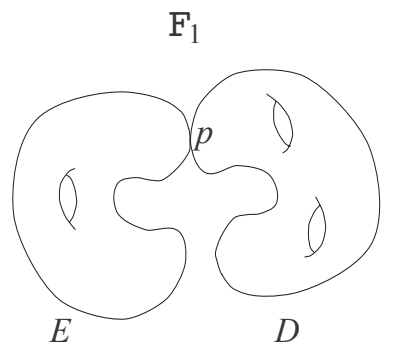

Fix the $j$-invariant and vary $p$ on $D$

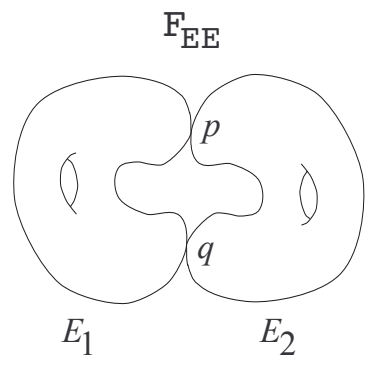

Fix the $j$-invariants and $q$ and vary $p$

FiguRE $1 . F_{1}$ and $F_{E E}$

Recall from [8, Theorem 1.1] that there is a divisorial contraction $T: \bar{M}_{g} \rightarrow \bar{M}_{g}^{p s}$ with exceptional locus $\Delta_{1}$ which replaces each elliptic tail with an ordinary cusp, where $\bar{M}_{g}^{p s}$ is the moduli space of pseudostable curves [17]. Also, recall from [2] that the cone $\mathrm{Nef}^{1}\left(\bar{M}_{3}\right)$ of nef divisors is generated by $\lambda, 12 \lambda-\delta_{0}$ and $10 \lambda-\delta_{0}-2 \delta_{1}$. Since $\delta_{1}$ is contracted by $T$, we deduce that $N e f^{1}\left(\bar{M}_{3}^{p s}\right)$ is generated by $10 \lambda^{p s}-\delta^{p s}$ and $12 \lambda^{p s}-\delta^{p s}$ : Indeed, since $\bar{M}_{3}^{p s}$ is of Picard number two, it suffices to show that these are nef and extremal. But these divisors pull back to the nef generators $10 \lambda-\delta_{0}-2 \delta_{1}$ and $12 \lambda-\delta_{0}$, and contract the loci $T\left(F_{E E}\right)$ and $T\left(F_{1}\right)$, respectively (Figure 1).

Let $B_{i} \subset \bar{M}_{3}$ denote the locus of curves obtained by taking the stabilization of the admissible cover of $C \in \bar{M}_{0,8}$ consisting of smooth rational curve $C_{1}$ with $i$ marked points and $C_{2}$ with $8-i$ marked points meeting in one node. These are divisors of the hyperelliptic locus $\bar{H}_{3} \subset \bar{M}_{3}$ that generate the rational Picard group of $\bar{H}_{3}$ (see, for instance, [4, P.302]). Examining the stabilizations of the admissible covers, we find that

(1) $B_{2}$ consists of irreducible curves with one node;

(2) $B_{3}$ consists of elliptic tails;

(3) $B_{4}$ consists of elliptic bridges.

Among these, $B_{3}$ is contracted by $T: \bar{M}_{3} \rightarrow \bar{M}_{3}^{p s}$ and $B_{4}$, by $\Psi: \bar{M}_{3}^{p s} \rightarrow \bar{M}_{3}^{c s}$. Hence the rational Picard group of the hyperelliptic locus $\bar{H}^{c s}$ of $\bar{M}_{3}^{c s}$ is generated by the image of $B_{2}$. The small contraction $\Psi^{+}: \bar{M}_{3}^{h s} \rightarrow \bar{M}_{3}^{c s}$ restricts to a small contraction on $\bar{H}^{h s}$, and $\left.\Psi^{+}\right|_{\bar{H}^{h s}}$ induces an isomorphism Pic $c_{\mathbb{Q}}\left(\bar{H}^{h s}\right) \simeq P i c_{\mathbb{Q}}\left(\bar{H}^{c s}\right)$. We conclude that $P i c_{\mathbb{Q}}\left(\bar{H}^{h s}\right)$ is generated by the image of $B_{2}$.

We summarize some results from $\S 2.1$ of [16]: Any smooth hyperelliptic curve of genus three is a divisor of type $(2,4)$ in $\mathbb{P}^{1} \times \mathbb{P}^{1}$. Let $F_{h}$ denote a pencil of these divisors, which is equivalent to twice the curve class $F_{h}^{\prime}$ in $B_{2}$ obtained by letting one of the six marked points move. Since $F_{h}^{\prime}$ completely misses the loci of elliptic tails and of elliptic bridges, the following intersection computation on $\overline{\mathcal{M}}_{3}$ carries over to 
$\bar{M}_{3}^{h s}$ :

$$
F_{h}^{\prime} \cdot \delta_{0}=14, \quad F_{h}^{\prime} \cdot \delta_{1}=0, \quad F_{h}^{\prime} \cdot \lambda=3 / 2 .
$$

It follows that $\left(28 \Lambda^{h s}-3 \Delta^{h s}\right) \cdot F_{h}^{\prime}=0$. Since $\operatorname{Pic}\left(\bar{H}^{h s}\right)$ is generated by the image of $B_{2}$, the divisor $28 \Lambda^{h s}-3 \Delta^{h s}$ is trivial on $h$. Therefore, $28 \Lambda^{h s}-3 \Delta^{h s}$ is nef and it contracts $\bar{H}^{h s}$ to a point.

Since the Mori cone of $\bar{M}_{3}^{h s}$ is of dimension two and $K_{\bar{M}_{3}^{h s}}+1 / 2 \bar{H}^{h s}+\frac{17}{28} \Delta^{h s}$ is big, the uniqueness of the extremal ray is obvious.

Proof of Theorem 1.(1). Given $\alpha \in\left(\frac{17}{28}, \frac{7}{10}\right) \cap \mathbb{Q}$ and small $\epsilon \in \mathbb{Q}_{+}, K_{\bar{M}_{3}^{h s}}+1 / 2 \bar{H}^{h s}+$ $\alpha \Delta^{h s}$ is a positive multiple of the linear combination

$$
(\alpha-17 / 28)\left(K_{\bar{M}_{3}^{h s}}+1 / 2 \bar{H}^{h s}+(7 / 10-\epsilon) \Delta^{h s}\right)+(7 / 10-\alpha-\epsilon)\left(K_{\bar{M}_{3}^{h s}}+1 / 2 \bar{H}^{h s}+(17 / 28) \Delta^{h s}\right) .
$$

Since $K_{\bar{M}_{3}^{h s}}+\bar{H}^{h s}+(7 / 10-\epsilon) \Delta^{h s}$ is ample and $K_{\bar{M}_{3}^{h s}}+1 / 2 \bar{H}^{h s}+(17 / 28) \Delta^{h s}$ is nef, $K_{\bar{M}_{3}^{h s}} 1 / 2 \bar{H}^{h s}+\alpha \Delta^{h s}$ is ample and $\bar{M}_{3}^{h s} \simeq \operatorname{Proj} \oplus_{n \geq 0} \Gamma\left(n\left(K_{\bar{M}_{3}^{h s}}+1 / 2 \bar{H}^{h s}+\alpha \Delta^{h s}\right)\right)$. Since $\delta_{1}$ is contracted by $T$, for any Cartier divisor $L$ on $\bar{M}_{3}^{p s}$ we have

$$
\Gamma\left(\bar{M}_{3}, T^{*} L\right)=\Gamma\left(\bar{M}_{3}^{p s}, T^{*} L+b \delta_{1}\right)=\Gamma\left(\bar{M}_{3}^{p s}, L\right), \quad \forall b \in \mathbb{Z}_{+} .
$$

Furthermore, since $\bar{M}_{3}^{p s}$ is isomorphic to $\bar{M}_{3}^{h s}$ away from a codimension two locus, $\Gamma\left(\bar{M}_{3}^{p s}, L\right)=\Gamma\left(\bar{M}_{3}^{h s}, L^{\prime}\right)$ for any reflexive sheaf $L$ on $\bar{M}_{3}^{p s}$ and the corresponding sheaf $L^{\prime}$ on $\bar{M}_{3}^{h s}$. Putting these all together, for $\alpha \in(17 / 28,7 / 10)$, we have:

$$
\begin{aligned}
\bar{M}_{3}(\alpha) & =\operatorname{Proj} \oplus_{n \geq 0} \Gamma\left(\bar{M}_{3}, n\left(K_{\bar{M}_{3}}+\alpha \delta\right)\right) \\
& =\operatorname{Proj} \oplus_{n \geq 0} \Gamma\left(\bar{M}_{3}, n\left(T^{*}\left(K_{\bar{M}_{3}^{p s}}^{p s}+\alpha \delta^{p s}\right)+(9-11 \alpha) \delta_{1}\right)\right. \\
& \simeq \operatorname{Proj} \oplus_{n \geq 0} \Gamma\left(\bar{M}_{3}^{p s}, n\left(K_{\bar{M}_{3}^{p s}}+\alpha \delta^{p s}\right)\right) \\
& \simeq \operatorname{Proj} \oplus_{n \geq 0} \Gamma\left(\bar{M}_{3}^{h s}, n\left(K_{\bar{M}_{3}^{h s}}+1 / 2 \bar{H}^{h s}+\alpha \Delta^{h s}\right)\right) \simeq \bar{M}_{3}^{h s} .
\end{aligned}
$$

2.2. The second divisorial contraction. This is the last (nontrivial) step in the $\log$ minimal program for $\bar{M}_{3}$. We have shown in Proposition 1 that $K_{\bar{M}_{3}^{h s}}+1 / 2 \bar{H}^{h s}+$ $(17 / 28) \Delta^{h s}$ contracts the hyperelliptic locus, and we aim to describe the resulting log canonical model $\bar{M}_{3}(17 / 28)$.

We first show that $\bar{M}_{3}(17 / 28)$ exists by using the base point freeness theorem. In general, if $(X, \Delta)$ is klt then so is $(X, a \Delta)$ for any $0<a<1$. Since $\left(\bar{M}_{3}^{h s}, 1 / 2 \bar{H}^{h s}+\right.$ $\left.\alpha \Delta^{h s}\right)$ is klt for any $\alpha \in(17 / 28,7 / 10),\left(\bar{M}_{3}^{h s}, a\left(1 / 2 \bar{H}^{h s}+\Delta^{h s}\right)\right)$ is klt for any $0<$ $a<7 / 10$. Choose $\epsilon$ such that $7 / 10-17 / 28<\epsilon<2(7 / 10-17 / 28)$. We have

(3) $2\left(K_{\bar{M}}^{h s}+1 / 2 \bar{H}^{h s}+(17 / 28) \Delta^{h s}\right)-\left(K_{\bar{M}}^{h s}+1 / 2 \bar{H}^{h s}+(7 / 10-\epsilon) \Delta^{h s}\right)=K_{\bar{M}_{3}^{h s}}+1 / 2 \bar{H}^{h s}+\beta \Delta^{h s}$ where $\beta:=2 \cdot 17 / 28-7 / 10+\epsilon$. The $\log$ canonical divisor (3) is nef and big since $17 / 28<\beta<7 / 10$. Recall Kawamata basepoint freeness: 
Theorem 2. [11, 3.3]: Let $(X, D)$ be a proper Kawamata log terminal pair with $D$ effective. Let $M$ be a nef Cartier divisor such that $a M-K_{X}-D$ is nef and big for some $a>0$. Then $|b M|$ has no basepoint for all $b \gg 0$.

Applying the theorem to $(X, D)=\left(\bar{M}_{3}^{h s}, 1 / 2 \bar{H}^{h s}+(7 / 10-\epsilon) \Delta^{h s}\right)$, and $(M, a)=$ $\left(K_{\bar{M}_{3}^{h s}}+1 / 2 \bar{H}^{h s}+(17 / 28) \Delta^{h s}, 2\right)$, we see that $\left|b\left(K_{\bar{M}_{3}^{h s}}+1 / 2 \bar{H}^{h s}+(17 / 28) \Delta^{h s}\right)\right|$ is base point free for $b \gg 0$. Hence

Lemma 1. $\bar{M}_{3}(17 / 28)$ exists as a projective variety, and there is a divisorial contraction

with exceptional locus $\bar{H}^{h s}$.

$$
\Theta: \bar{M}_{3}^{h s} \rightarrow \bar{M}_{3}(17 / 28)
$$

We recall some classical results on the GIT of plane quartics [14], [1]. Consider the natural action of $P G L_{6}$ on the space $\mathbb{P}\left(\Gamma\left(\mathcal{O}_{\mathbb{P}^{2}}(4)\right)\right)$ of plane quartics. With respect to this action, a plane quartic curve $C$ is

(i) stable if and only if it has ordinary nodes and cusps as singularity;

(ii) strictly semistable if it is a double conic or has a tacnode. Moreover, $C$ belongs to a minimal orbit if and only if it is either a double conic or the union of two tangent conics (where at least one is smooth).

The minimal orbit statement in (2) implies that in the GIT quotient space $\bar{Q}=$ $\mathbb{P}\left(\Gamma\left(\mathcal{O}_{\mathbb{P}^{2}}(4)\right)\right) / / P G L_{6}$, an irreducible tacnodal curve is identified with the corresponding cat-eye, as in $\bar{M}_{3}^{h s}$.

Proof of Theorem 1.(2). Consider the universal quartic curve $\mathcal{X}$ :

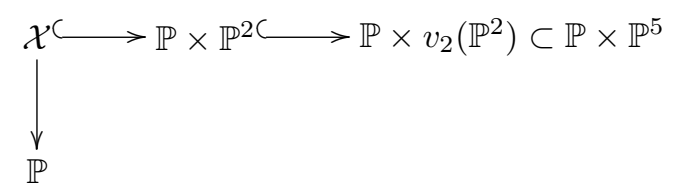

where $\mathbb{P}:=\mathbb{P}\left(\Gamma\left(\mathcal{O}_{\mathbb{P}^{2}}(4)\right)\right)^{s s}$ and $v_{2}$ denotes the second Veronese embedding. Abusing notation, let $\mathcal{X}$ also denote the image of $\mathcal{X}$ in $\mathbb{P} \times \mathbb{P}^{5}$. Let $y \in \bar{Q}$ denote the point corresponding to the double conic. Away from the orbit $O\left(\mathcal{X}_{y}\right)$ of the double conic $\mathcal{X}_{y}, \mathcal{X} \hookrightarrow \mathbb{P} \times \mathbb{P}^{5}$ is a family of bicanonical h-semistable curves, and induces a map from $\mathbb{P} \backslash O\left(\mathcal{X}_{y}\right)$ to $H i l b_{3,2}^{s s}$ and subsequently to the quotient $H i l b_{3,2} / / S L$. This map is $P G L_{3}$ invariant, and it descends to give $f: \bar{Q} \backslash\{y\} \rightarrow \bar{M}_{3}^{h s}$. Recall that $Z^{+}$denotes the locus of tacnodal curves in $\bar{M}_{3}^{h s}$, and let $Z^{q}$ denote the locus of tacnodal curves in $\bar{Q}$. Over the stable locus, we have isomorphisms

$$
\bar{Q} \backslash\left(\{y\} \cup Z^{q}\right) \simeq \bar{M}_{3}^{h s} \backslash\left(Z^{+} \cup \bar{H}^{h s}\right) \simeq \bar{M}_{3} \backslash\left(\Delta_{1} \cup\{\text { elliptic bridges }\} \cup \bar{H}_{3}\right)
$$

where $f$ induces the first isomorphism. The loci $Z^{q}$ and $Z^{+}$are of codimension $\geq 2$, and $\left.f\right|_{Z^{q}}$ is bijective (see item (ii) above and the subsequent remark). It follows that the inverse rational map $f^{-1}$ is regular on $Z^{+}$, giving an isomorphism

$$
\bar{Q} \backslash\{y\} \simeq \bar{M}_{3}^{h s} \backslash \bar{H}^{h s} \simeq \bar{M}_{3}(17 / 28) \backslash\left\{\Theta\left(\bar{H}_{3}\right)\right\} .
$$

The assertion now follows by applying Hartogs' Lemma again. 
Proof of Theorem 1.(3) (4). Let $\lambda^{\bar{Q}}$ and $\delta^{\bar{Q}}$ denote the unique divisors on $\bar{Q}$ extend$\operatorname{ing} \Lambda^{h s}$ and $\Delta^{h s}$ on the open set $\bar{Q} \backslash\{y\} \simeq \bar{M}_{3}^{h s} \backslash Z^{+}$. We have

$$
K_{\bar{Q}}+5 / 9 \delta^{\bar{Q}}=13 \lambda^{\bar{Q}}-2 \delta^{\bar{Q}}+5 / 9 \delta^{\bar{Q}}=(13 / 9-2+5 / 9) \delta^{\bar{Q}}=0,
$$

that is, $K_{\bar{Q}}+5 / 9 \delta^{\bar{Q}}$ is trivial and $\bar{M}_{3}(5 / 9) \simeq \operatorname{Proj} \oplus_{n \geq 0} \Gamma\left(n\left(K_{\bar{Q}}+5 / 9 \delta^{\bar{Q}}\right)\right)$ is a point, where the isomorphism can be obtained by the same argument used in (2) of the preceding section. It also follows from (4) that $K_{\bar{Q}}+\alpha \delta^{\bar{Q}}$ is ample for $\alpha \in(5 / 9,17 / 28]$ since it is a linear combination

$$
a\left(K_{\bar{Q}}+5 / 9 \delta^{\bar{Q}}\right)+b\left(K_{\bar{Q}}+17 / 28 \delta^{\bar{Q}}\right)
$$

for some positive rational numbers $a, b$ (determined by $\alpha$ ).

\section{Relation to other moduli spaces: Work of Hassett, Hacking and Kondo}

There are various constructions of compact moduli spaces of plane quartics [5], [3], [12]. How do they fit in our minimal model program? In this section, we sketch these moduli spaces and show that they are $\log$ canonical models for $\bar{M}_{3}$. In fact, Hassett already showed in [5] that his moduli space is isomorphic to $\bar{M}_{3}$, but we give a brief description of his moduli space here as we need it to identify other moduli spaces.

3.1. Hassett's moduli space $\overline{\mathfrak{P}}_{4}$. In [5], B. Hassett constructed a compact moduli space $\overline{\mathcal{P}}_{4}$ of plane curves of degree 4 by using smoothable stable log surfaces. There is a forgetting morphism $F: \overline{\mathfrak{P}}_{4} \rightarrow \bar{M}_{3}$ defined by $F((S, C))=C$ which in fact, is an isomorphism: For each curve $C$ in $\bar{M}_{3}$, Hassett explicitly constructs the unique corresponding surface $S$ with $(S, C) \in \overline{\mathfrak{P}}_{4}$. Then he shows that the morphism $F$ is proper, birational, and locally an isomorphism.

The cone of effective divisors $\overline{N E}^{1}\left(\overline{\mathcal{M}}_{3}\right)$ is generated by $\delta_{0}, \delta_{1}$ and $h$. A general element in each of these divisors corresponds to the following stable log surface in $\overline{\mathfrak{P}}_{4}$ :

(1) $C$ in $\delta_{0} \Longleftrightarrow\left(\mathbb{P}^{2}, C\right)$;

(2) $C=C_{1} \cup_{p} C_{2}$ in $\delta_{1}$ where $C_{1}$ and $C_{2}$ are irreducible curves of genus two and one respectively $\Longleftrightarrow\left(S_{1} \cup_{B} S_{2}, C\right)$ where $S_{1}$ is the toroidal blowup of $\mathbb{P}^{2}$ and $S_{2}=\mathbb{P}(1,2,3)$. The curve $C_{1}$ in $S_{1}$ does not pass through the two singular points of type $\frac{1}{2}(1,1)$ and $\frac{1}{3}(1,1)$ of the surface $S_{1}$. Also the curve $C_{2}$ in $S_{2}$ does not pass through the two singular points $\frac{1}{2}(1,1)$ and $\frac{1}{3}(1,2)$ of the surface $S_{2}$. The curve $C=C_{1} \cup_{p} C_{2}$ meets the double curve $B$ at the point $p$.

(3) $C$ in $h \Longleftrightarrow(S, C)$ where $S=\mathbb{P}(1,1,4)$. Since $C$ is a smooth hyperelliptic curve of genus three, $C$ is regarded as a bisection of the rational surface $\mathbb{F}_{4}=\mathbb{P}\left(\mathcal{O}_{\mathbb{P}^{1}} \oplus \mathcal{O}_{\mathbb{P}^{1}}(4)\right) . S$ is obtained by the contraction of the zero section. The curve $C$ does not pass through the singular point of type $\frac{1}{4}(1,1)$. 
3.2. Hacking's moduli space $\overline{\mathfrak{P}}_{4}^{\prime}$. Hacking gave an alternate compactification of the moduli space of plane curves of degree $d[3]$ by employing a method similar to [5] but allowing worse singularities. We denote his moduli space for $d=4$ by $\overline{\mathfrak{P}}_{4}^{\prime}$ (His original notation $\mathcal{M}_{3}$ is unfortunately reserved for the moduli stack of smooth curves of genus three.) A stable pair of degree 4 is a pair consisting of a surface $S$ and a curve $C \subset S$ such that $(S, \beta C)$ has semi-log canonical singularities and $K_{S}+\beta C$ is ample, where $\beta$ is a rational number $\frac{3}{4}+\epsilon$ for a sufficient small positive number $\epsilon$. We also impose the condition that there be a one parameter family of deformations $(\mathcal{S}, \mathcal{C})$ whose general fiber is a smooth pair $\left(\mathbb{P}^{2}\right.$, smooth quartic curve). Both $\mathbb{Q}$-divisors $K_{\mathcal{S}}+\beta \mathcal{C}$ and $\mathcal{C}$ are $\mathbb{Q}$-Cartier.

From his classification of stable surfaces of degree $4, \overline{\mathfrak{P}}_{4}^{\prime}=Z_{0} \cup Z_{1} \cup Z_{2}$ where $Z_{1}$ has codimension 1 and $Z_{2}$ has codimension 2 such that:

(1) Any element in $Z_{0}$ is a pair $\left(\mathbb{P}^{2}, C\right)$ where $C$ is a pseudo-stable plane curve of degree 4;

(2) Any element in $Z_{1}$ is a pair $(S, C)$ where $S=\mathbb{P}(1,1,4)$ and $C$ is a (degenerating) hyperelliptic curve of genus 3 . The curve $C$ does not pass through the singular point of type $\frac{1}{4}(1,1)$;

(3) Any element in $Z_{2}$ is a pair $(S, C)$ where $S=S_{1} \cup_{B} S_{2}$ is the union of two $\mathbb{P}(1,1,2)$ s and $C$ is a (degenerating) elliptic bridge. Both irreducible components $S_{1}$ and $S_{2}$ have cyclic quotient singularities of type $\frac{1}{2}(1,1)$ on the double curve $B=\mathbb{P}^{1}$. The curve $C$ does not pass through the singular points of $S_{1}$ and $S_{2}$.

If $(S, C)$ is a stable pair in $\overline{\mathfrak{P}}_{4}^{\prime}$ then $C$ has nodes and cusps as singularities, and there is a forgetting morphism $F^{\prime}: \overline{\mathfrak{P}}_{4}^{\prime} \rightarrow \bar{M}_{3}^{p s}$ defined by $F^{\prime}((S, C))=C$.

3.3. $\overline{\mathfrak{P}}_{4}$ and $\overline{\mathfrak{P}}_{4}^{\prime}$ as log canonical models. Hassett proved that $\overline{\mathfrak{P}}_{4}$ is isomorphic to the moduli space $\bar{M}_{3}$ of stable curves. Implicit in Hacking's work is that

Proposition 2. $\overline{\mathfrak{P}}_{4}^{\prime}$ is isomorphic to $\bar{M}_{3}(9 / 11)$.

Proof. It is remarked in Hassett's paper that for $\beta>5 / 6, K_{S}+\beta C$ is ample for any pair $(S, C) \in \overline{\mathfrak{P}}_{4}$. Therefore $\overline{\mathfrak{P}}_{4}(\beta) \simeq \overline{\mathfrak{P}}_{4}$ for $\beta>5 / 6$, where $\overline{\mathfrak{P}}_{4}(\beta)$ denotes the moduli space of pairs constructed in $\S 3.2$ using the prescribed value of $\beta$ whereas $3 / 4+\epsilon$ was used for $\beta$ in $§ 3.2$.

We consider what happens at $\beta=5 / 6$. First, there is a morphism $T^{\prime}: \overline{\mathfrak{P}}_{4} \rightarrow$ $\overline{\mathfrak{P}}_{4}(5 / 6)$ that associates to $(S, C)$ the pair $\left(S^{\prime}, C^{\prime}\right)$ constructed as follows: By assumption $(S, C)$ is smoothable and there is a one-parameter family $(\mathcal{S}, \mathcal{C}), \pi: \mathcal{S} \rightarrow$ $\operatorname{Spec}(k[[t]])$ whose special fibre is $(S, C)$ and whose generic fibre is smooth. $S^{\prime}$ is then the special fibre of the relative $\log$ canonical model $\mathcal{S}^{\prime}:=$ Proj $\oplus_{m \geq 0} \pi_{*}\left(m\left(K_{\mathcal{S}}+\right.\right.$ $5 / 6 \mathcal{C})$ ) and $\mathcal{C}^{\prime}$, the scheme theoretic image $f\left(\mathcal{C}^{\prime}\right)$ where $f$ is the canonical fibration from $\mathcal{S}$ to $\mathcal{S}^{\prime}$. Regardless of the choice of the smoothing, $\left(S^{\prime}, C^{\prime}\right)$ is always the log canonical model of $(S, C)$ (If $S$ has more than one component, then $S^{\prime}$ is the union of the log canonical models of the components with the restriction of the log canonical divisor as boundary divisor.)

Since the divisor $K_{S}+5 / 6 C$ is ample for all pairs $(S, C) \in \overline{\mathfrak{P}}_{4}$ except the ones with $S=$ (toroidal blowup of $\left.\mathbb{P}^{2} \cup \mathbb{P}(1,2,3)\right)$ and $C$ an elliptic tail, $T^{\prime}$ is an isomorphism 
away from the locus $\mathcal{D}_{1} \subset \overline{\mathfrak{P}}_{4}$ of elliptic tails. For elliptic tails $\left(S_{1} \cup_{B} S_{2}, C\right)$, restricting $K_{S}+5 / 6 C$ on $S_{2}$, we find that

$$
\left.\left(K_{S}+\frac{5}{6} C\right)\right|_{S_{2}}=K_{S_{2}}+\frac{5}{6} C+B=\mathcal{O}_{S_{2}}(-6+5+1)=\mathcal{O}_{S_{2}} .
$$

This means that $f: S \rightarrow S^{\prime}$ contracts the elliptic component: In fact, Hassett proves that $f: C \rightarrow C^{\prime}$ replaces the elliptic tail by an ordinary cusp. Also, the log canonical models of $(S, C) \in \overline{\mathfrak{P}}_{4}$ with respect to $K_{S}+5 / 6 C$ are precisely the stable pairs in Hacking's moduli space. All in all, we have a birational contraction

$$
T^{\prime}: \bar{M}_{3} \rightarrow \overline{\mathfrak{P}}_{4}^{\prime}
$$

such that $T^{\prime}$ (curve) $=$ point if and only if curve $\subset \bar{M}_{3}$ is a curve in $\delta_{1}$ obtained by varying the $j$-invariant of the elliptic tail. Hence the forgetful map $F^{\prime}: \overline{\mathfrak{P}}_{4}^{\prime} \rightarrow \bar{M}_{3}^{p s}$ is a bijective birational morphism between normal varieties. By Zariski's main theorem, $F^{\prime}$ is an isomorphism.

3.4. Kondo's compact moduli space. Kondo constructed a compact moduli space of plane quartic curves by using the period domains of K3 surfaces [12]. Let $C$ be a smooth plane quartic curve. Then the cyclic $\mathbb{Z}_{4}$-cover of $\mathbb{P}^{2}$ branched along $C$ is a K3 surface. The period domains of such K3 surfaces correspond to an arithmetic quotient of a bounded symmetric domain $\mathcal{D}$ minus two hyperplanes. He extends this correspondence to the whole $\mathcal{D}$ by allowing hyperelliptic curves of genus 3 and singular pseudo-stable plane curves of genus 3 [12]. By using the Baily-Borel compactification of period domains, he constructs a compact moduli space which is normal and whose boundary is one point. Details can be found in [1] and [12]. Let us denote Kondo's compact moduli space by $\overline{\mathfrak{K}}$, and denote the unique point in the boundary by $q$.

Proposition 3. $\overline{\mathfrak{K}} \simeq \bar{M}_{3}\left(\frac{7}{10}\right)$.

Proof. Recall that $C h o w_{3,2} / / S L_{6}$ is isomorphic to $\bar{M}_{3}\left(\frac{7}{10}\right)$ and all strictly semistable points are identified in $\mathrm{Chow}_{3,2} / / S L_{6}$. Considering Kondo's construction and the classification of curves in $C h o w_{3,2} / / S L_{6}$ reveals that there is a forgetful morphism $F_{\overline{\mathfrak{K}}}: \overline{\mathfrak{K}} \rightarrow$ Chow $_{3,2} / / S L$ mapping $(S, C)$ to $C$.

We claim that there is a birational map $\overline{\mathfrak{P}}_{4}^{\prime} \rightarrow \overline{\mathfrak{K}}$ that induces an isomorphism

$$
\overline{\mathfrak{P}}_{4}^{\prime} \backslash Z_{2} \simeq \overline{\mathfrak{K}} \backslash\{q\} \text {. }
$$

Recall that $\overline{\mathfrak{P}}_{4}^{\prime}=Z_{0} \cup Z_{1} \cup Z_{2}$. Let $\left(\mathbb{P}_{Z_{0}}^{2}, \mathcal{C}\right)$ denote the universal pair over $Z_{0}$. The universal pair has $\mathbb{P}^{2}$ as the constant surface part and is parametrized by the curve part that walks through all pseudostable plane curves. By taking the cyclic $\mathbb{Z}_{4}$-cover of $\mathbb{P}^{2}$ branched along the curve, we obtain a universal pair $(\mathcal{X}, \mathcal{C})$ of $\mathrm{K} 3$ surfaces paired with pseudo-stable plane curves. This induces a morphism from $Z_{0}$ to $\overline{\mathfrak{K}}$. It is an isomorphism onto its image since it is a bijective morphism of normal varieties. A similar construction gives an isomorphism from $Z_{1}$ to its image in $\overline{\mathfrak{K}}$. Considering the description of curves in $Z_{0}$ and $Z_{1}$, we obtain the desired isomorphism $\overline{\mathfrak{P}}_{4}^{\prime} \backslash Z_{2} \simeq \overline{\mathfrak{K}} \backslash\{q\}$. 
The upshot of the isomorphism (5) is that, since $\overline{\mathfrak{P}}_{4}^{\prime} \backslash Z_{2} \simeq \bar{M}_{3}^{p s} \backslash Z$ which is isomorphic under $\Psi$ to $\bar{M}_{3}^{c s} \backslash\{$ strictly semistable point $\}$, we have

$$
\overline{\mathfrak{K}} \backslash\{q\} \simeq\left(\text { Chow }_{3,2} / / S L_{6}\right) \backslash\{\text { strictly semistable point }\} .
$$

By Hartog's theorem this birational map defined away from a locus of codimension $>2$ is extended to an isomorphism since both varieties are normal.

Remark 1. To our knowledge, the moduli space $\bar{M}_{3}^{h s}$ of h-stable curves is a new modular compactification of $M_{3}$. But we note that h-stable curves are precisely the curves that appear in the semistable pairs of degree four [3]. Although Hacking defined the notion, the corresponding moduli space was not constructed.

\section{References}

[1] M. Artebani, The moduli space of genus three curves as a period domain of K3 surfaces, Thesis, Universitá degli Studi di Genova (2005)

[2] C. Faber, Chow rings of moduli spaces of curves. I. The Chow ring of $\bar{M}_{3}$, Ann. of Math. (2) 132 (1990), no. 2, 331-419.

[3] P. Hacking, Compact moduli of plane curves, Duke Math. J. 124 (2004), no. 2, 213-257.

[4] J. Harris and I. Morrison, Moduli of curves, Vol. 187 of Graduate Texts in Mathematics, Springer-Verlag, New York (1998), ISBN 0-387-98438-0; 0-387-98429-1.

[5] B. Hassett, Stable log surfaces and limits of quartic plane curves, Manuscripta Math. 100 (1999), no. 4, 469-487.

[6] — Classical and minimal models of the moduli space of curves of genus two, in Geometric methods in algebra and number theory, Vol. 235 of Progress in mathematics, 160-192, Birkhäuser, Boston (2005).

[7] B. Hassett and D. Hyeon, Log minimal model program for the moduli space of curves: the first flip (2008). ArXiv:0806.3444v1 [math.AG].

[8] — Log canonical models for the moduli space of curves: the first divisorial contraction, Trans. Amer. Math. Soc. 361 (2009), no. 8, 4471-4489.

[9] D. Hyeon and Y. Lee, Stability of tri-canonical curves of genus two, Math. Ann. 337 (2007), no. $2,479-488$

[10] - Stability of bicanonical curves of genus three, J. Pure Appl. Algebra 213 (2009), no. 10, 1991-2000.

[11] J. Kollár and S. Mori, Birational geometry of algebraic varieties, Vol. 134 of Cambridge Tracts in Mathematics, Cambridge University Press, Cambridge (1998), ISBN 0-521-63277-3. With the collaboration of C. H. Clemens and A. Corti, Translated from the 1998 Japanese original.

[12] S. Kondō, A complex hyperbolic structure for the moduli space of curves of genus three, J. Reine Angew. Math. 525 (2000) 219-232.

[13] A. Moriwaki, Relative Bogomolov's inequality and the cone of positive divisors on the moduli space of stable curves, J. Amer. Math. Soc. 11 (1998), no. 3, 569-600.

[14] D. Mumford, Geometric invariant theory, Ergebnisse der Mathematik und ihrer Grenzgebiete, Neue Folge, Band 34, Springer-Verlag, Berlin (1965).

[15] — Stability of projective varieties, Enseignement Math. (2) 23 (1977), no. 1-2, 39-110.

[16] W. Rulla, The birational geometry of $\bar{M}_{3}$ and $\bar{M}_{2,1}$ (2001). Ph. D. Thesis, University of Texas at Austin.

[17] D. Schubert, A new compactification of the moduli space of curves, Compositio Math. 78 (1991), no. 3, 297-313.

Department of Mathematics, Postech, Pohang, Gyungbuk, 790-784, Republic of Korea E-mail address: dhyeon@postech.ac.kr 
Department of Mathematics, Sogang Univeristy, Sinsu-dong, Mapo-gu, Seoul 121-742, REPUblic of Korea

E-mail address: ynlee@sogang.ac.kr 